\title{
Diabetes and Hypertension Among Patients with Psychiatric Illnesses Attending Outpatient Services in a Tertiary Mental Health Centre: A Cross-sectional Study from South India
}

\author{
RamakrishnanParvathy, BalasankaralingamSugetha, RamachandranPadmavati ${ }^{\star}$
}

\begin{abstract}
Background: High burden of comorbid physical noncommunicable diseases (NCDs) is observed in patients with psychiatric illnesses. However, little attention is paid to physical illnesses of this patient population. This study evaluated the self-reported frequency of physical NCDs in patients with psychiatric illnesses, current involvement of psychiatrists in managing these NCDs and association of NCDs with socio-demographic and clinical factors.
\end{abstract}

Materials and Methods: This cross-sectional study was conducted in out-patient department of a tertiary mental health centre in southern India. Consecutive adult patients $(\mathrm{N}=450)$ fulfilling the study's inclusion criteria were interviewed regarding comorbid physical NCDs including diabetes and hypertension, and aspects related to their diagnosis and treatment. Psychiatric diagnoses and psychotropic treatment information was obtained from electronic patient records maintained at the centre.

Results: Overall frequency of any physical NCD was $24.9 \%$, with diabetes and hypertension reported by $15.8 \%$ and $8.7 \%$ of patients, respectively. Patients with diabetes or hypertension were more likely to be older, have a family history of diabetes/hypertension, or psychiatric illness for at least 5 years or longer. Less than $8 \%$ of patients with diabetes or hypertension were treated for their physical NCD by psychiatrists. Forty patients with elevated BP $(\geq 140$ and/or $\geq 90 \mathrm{mmHg}$ ) or at risk for hypertension were identified while conducting physical examination as part of the study.
Conclusion: Diabetes and hypertension are common physical NCDs in patients with psychiatric illness. Therefore, it is important that psychiatrists be aware of and identify patients who are at risk for such NCDs.

Keywords: Psychiatric Disorders, Physical Disorders, Non-Communicable Diseases, Diabetes, Hypertension, Frequency

Running Title: Physical comorbidities in psychiatric outpatients

Address for Correspondence: Dr. Padmavati Ramachandran, Director, Schizophrenia Research Foundation, Anna Nagar West Extension, Chennai, Tamilnadu, India; E-mail: padmavati@scarfindia.org

How to Cite this Article: Parvathy R, Sugetha B, Padmavati R. Diabetes and Hypertension among patients with psychiatric illnesses attending outpatient services in a tertiary mental health centre: A crosssectional study from South India. Indian Journal of Mental Health and NeuroSciences.2020;3(1):pp 15-22

There is a large body of evidence demonstrating that patients with mental illnesses have greater physical health morbidity, ${ }^{1,2,3}$ with $60 \%$ of premature deaths amongst these patients attributed to physical illnesses such as cardiovascular and pulmonary diseases. ${ }^{4}$ Furthermore, patients with severe mental illnesses have a shorter lifespan compared to the general population. ${ }^{5,6}$ The increase in morbidity and mortality in patients with schizophrenia is partly explained by a higher prevalence of modifiable risk factors such as

* Schizophrenia Research Foundation, Anna Nagar West Extension, Chennai, Tamilnadu, India 
lack of exercise, obesity, alcohol misuse, smoking, and unemployment. ${ }^{7,8}$ While these factors are related to the patient and their lifestyle choices, there also differences in and concerns around the access, utilization and provision of quality health care that patients with mental illnesses receive for their physical health. This contributes to poor physical health outcomes in this group of patients. ${ }^{9}{ }^{10,11} \mathrm{With}$ respect to medications given to treat psychiatric illnesses, second generation antipsychotics likely alter glucose metabolism and cause weight gain, thereby worsening the risk for metabolic syndrome (MetS) and cardiovascular diseases in these patients. ${ }^{12,13}$ Finally, a major health system-specific issue such as the fragmentation between mental health and primary health care has exacerbated the growing problem of physical comorbidities and premature mortality in patients with mental illnesses. ${ }^{4}$

The Clinical Antipsychotic Trials of Intervention Effectiveness (CATIE) study examined the effectiveness of different antipsychotic medications in over 1400 patients with schizophrenia across different sites. ${ }^{10}$ Apart from measuring psychiatric outcomes, this study also assessed physical health indicators. Diabetes was seen in $11 \%$ of patients, while MetS and impaired fasting glucose were observed in $40 \%$ and $25 \%$ of patients, respectively. The most important finding of the CATIE study, apart from revealing at least $40 \%$ or higher rates of untreated diabetes, hypertension or hyperlipidemia, was that psychiatrists tended to ignore life-threatening, treatable medical conditions in patients presenting for treatment with schizophrenia. These results highlight the need for increased attention and basic monitoring of cardiovascular risk factors in this psychiatric population. ${ }^{10}$

A few studies in India have measured the prevalence and correlates of physical comorbidities such as diabetes, hypertension and/or MetS in patients with mental illnesses being treated at tertiary psychiatric centers. MetS was prevalent in 35\%-38\% of psychiatric inpatients in a tertiary care center in northern India, a rate higher than that observed in the general population. ${ }^{14,15}$ In an out-patient psychiatric facility, hypertension and diabetes were amongst the most common physical illnesses with prevalence rates of $29.1 \%$ and $10 \%$, respectively. ${ }^{16}$ In a study in Assam, almost $30 \%$ of patients with schizophrenia presented with MetS, and risk factors for developing MetS were female gender, smoking, family history of chronic
NCDs and use of atypical antipsychotics. ${ }^{17}$ However, these studies had several limitations such as a smaller sample size ${ }^{14,17}$, or significant number of patients dropping out of the study or unwilling to participate. ${ }^{16}$

It is widely agreed that lifestyle risk factors largely explain the excess morbidity and mortality in persons with mental illness. However, it is noted all over the world, that screening and assessment of physical health problems remains poor, even in developed countries. A crowded mental health services often does not provide the necessary opportunity to screen and assess physical health conditions by a mental health team. However, a simple screening and history of physical health conditions can provide valuable information to monitoring and treatment of crucial physical parameters.

In our study, we aimed to evaluate the frequency of physical non-communicable diseases (NCDs) such as diabetes, hypertension, chronic lung disease, heart disease and chronic kidney disease, as elicited from information gathered among patients with psychiatric disorders at an out-patient mental health center and measures of anthropometric and blood pressure indices. In addition, we evaluated the extent to which psychiatrists at the out-patient mental health centerelicited information and participated in management of physical comorbidities in this patient population. Finally, we assessed the association of these physical NCDs with socio-demographic and clinical factors, psychiatric diagnoses, and psychotropic medications.

\section{MATERIAL \& METHODS}

Study site, design and participants: The current study was conducted among patients seeking psychiatric care at the out-patient facility of the Schizophrenia Research Foundation (SCARF) in Chennai, India. It was a crosssectional observational study, carried out between July 2017 and November 2017.

Male and female patients aged 18 years and above with known psychiatric illness and being treated at SCARF were consecutively selected for the study during their visit. Those who consented to be part of the study were included, irrespective of the type of psychiatric diagnosis, duration of psychiatric disease, type of comorbid physical $\mathrm{NCD}(\mathrm{s})$ or use of psychotropic medications. 
Sample size estimation: We used the method outlined by Naing et al. to estimate sample size. ${ }^{18}$ The sample size was estimated using the formula: $\mathrm{n}=\mathrm{Z} 2^{\star} \mathrm{P}^{\star}(1-\mathrm{P}) / \mathrm{d}^{2}$, where $\mathrm{n}$ is sample size, $\mathrm{Z}$ is $\mathrm{Z}$ statistic for a level of confidence (we used $Z$ value of 1.96 for $95 \%$ confidence level), $P$ is the expected prevalence or proportion (in proportion of one; if $20 \%, \mathrm{P}=0.2$ ), and $\mathrm{d}$ is precision (in proportion of one; if $5 \%, d=0.05$ ). Based on a pilot study of 91 patients, we estimated prevalence of atleast $1 \mathrm{NCD}$ to be $30.8 \%$. Using this estimate in the above formula, we obtained a sample size of 328 patients.

Data collection: A survey questionnaire was developed based on the WHO STEP-wise approach to surveillance methodology, a standardized method to collect, analyze and disseminate data on risk factors for NCDs, especially in low-to-middle income countries. ${ }^{19}$ The questionnaire included socio-demographic characteristics, current psychiatric diagnoses, duration of psychiatric disease, use of psychotropic medications, known medical history of physical NCDs such as hypertension, diabetes mellitus, heart disease, chronic lung disease, or renal disease, details on how/where these comorbidities were diagnosed, pharmacological management of these comorbidities and the associated treatment duration. Further, information on family history of psychiatric illness or physical NCDs and lifestyle such as diet modifications, physical activity, smoking and alcohol intake were collected. Anthropometric measurements such as height, weight, waist and hip circumference were performed using a non-stretchable tape. Blood pressure (BP) was measured in the right arm using standard cuffs fitted with a mercury sphygmomanometer in sitting position.

Patients were interviewed in the local language by a trained medical doctor and responses to the interview were patient-reported or caregiver-reported in case the patient was absent. Psychiatric diagnoses of the patients were obtained from their electronic medical records available at the outpatient center. These diagnoses had been previously recorded by the treating psychiatrist at the time of patient registration and treatment initiation. Diagnostic categories were identified by the treating psychiatrist based on ICD-10 codes F01-F99 and used for analysis..$^{20}$ Psychotropic medications prescribed to the patients were recorded based on their prescriptions. For the analysis, these medications were classified into four categories, namely patients taking: (1) one antipsychotic (either first- or second-generation); (2) more than one type of antipsychotic; (3) antipsychotic medications combined with other psychotropic medications (eg. benzodiazepines, mood stabilizers, anti-depressant drugs, anti-epileptics drugs, anticholinergics); and (4) other medicines or combinations which do not fall in the first three categories (eg. Combinations based on mood stabilizers, benzodiazepines and/or anti-depressants).

Data analysis: STATA (release 15.1, Texas, USA: Stata Corp) was used for all statistical analyses. Statistical differences in the socio-demographic and clinical characteristics between patients with and without diabetes/hypertension were tested using Chi-square test or Fisher's exact test (as appropriate) for categorical data and Student's t-test or Mann-Whitney test (as appropriate) for continuous data. Similarly, the association of diabetes/hypertension with different psychiatric diagnoses and the use of various psychotropic medications were tested using a Chi-square test or Fisher's exact test (as appropriate). A p-value less than 0.05 was considered statistically significant.

Ethics: The study obtained all required ethical approvals from the Institutional Review Board at SCARF and informed consent was taken individually from all participants.

\section{RESULTS}

Sociodemographic and clinical characteristics of the study population

A total of 450 patients fulfilling the inclusion criteria were included in the analysis. As shown in Table 1, mean age of the entire sample population was 40.8 years, with mean age comparable between males and females. Males represented $44 \%$ of the study population. Mean body mass index (BMI) and mean blood pressure (BP) of the study population was $27.3 \mathrm{~kg} / \mathrm{m} 2$ and $118.8 / 74.7 \mathrm{mmHg}$, respectively. A large proportion of patients did not smoke (90.9\%) or consume alcohol (95.8\%). Approximately one-third of the patients had a family history of psychiatric illnesses (32.7\%), while $63.3 \%$ of patients had a family history of diabetes/ hypertension (Table 1 ). 
Table 1. Demographic characteristics and information on physical illness of the study population

\begin{tabular}{|l|c|c|c|}
\hline Characteristic & $\begin{array}{c}\text { Overall } \\
\mathbf{N}=\mathbf{4 5 0}\end{array}$ & $\begin{array}{c}\text { Male } \\
\mathbf{N = 1 9 8}\end{array}$ & $\begin{array}{c}\text { Female } \\
\mathbf{N = 2 5 2}\end{array}$ \\
\hline Age, mean \pm SD (years) & $40.8 \pm 12.2$ & $39.8 \pm 12.2$ & $41.7 \pm 12.1$ \\
\hline Family history of any physical NCD & $285(63.3)$ & $122(61.6)$ & $163(64.7)$ \\
\hline Smoking & $46(10.1)$ & $46(23.2)$ & $0(0)$ \\
\hline Alcohol & $19(4.2)$ & $16(8.1)$ & $3(1.2)$ \\
\hline BMI, mean $\pm \mathrm{SD}\left(\mathrm{kg} / \mathrm{m}^{2}\right)^{\S}$ & $27.3 \pm 5.1$ & $25.8 \pm 4.4$ & $28.2 \pm 5.2$ \\
\hline SBP, mean $\pm \mathrm{SD}(\mathrm{mmHg})^{\#}$ & $118.8 \pm 16.0$ & $121.9 \pm 16.7$ & $116.5 \pm 15.1$ \\
\hline DBP, mean $\pm \mathrm{SD}(\mathrm{mmHg})^{\#}$ & $74.7 \pm 10.8$ & $77.4 \pm 11.9$ & $72.7 \pm 9.3$ \\
\hline Diabetes & $71(15.8)$ & $27(13.6)$ & $44(17.5)$ \\
\hline Hypertension & $39(8.7)$ & $11(5.6)$ & $28(11.1)$ \\
\hline Diabetes and hypertension & $20(4.4)$ & $5(2.5)$ & $15(5.9)$ \\
\hline
\end{tabular}

(All data expressed as $\mathrm{n}(\%)$, unless otherwise indicated. ${ }^{\$}$ Overall $\mathrm{N}=311$, Males $\mathrm{N}=124$, Females $\mathrm{N}=187$ as data on height and weight were missing from some patients; ${ }^{*}$ Overall $\mathrm{N}=400$, Males $\mathrm{N}=171$, Females $\mathrm{N}=229$ )

Majority (62.2\%) of patients had their psychiatric illness for at least 5 years or longer, with $63.8 \%$ of patients being treated for less than 5 years at SCARF. Upon classifying patients based on their psychiatric diagnoses, $64.4 \%$ had schizophrenia, schizotypal and delusional disorders, 21.2\% with mood (affective) disorders and 5.8\% had neurotic, stress-related and somatoform disorders. Majority of patients (68.6\%) in the entire sample population were taking combinations of antipsychotics with other psychotropic medications, $14.3 \%$ were prescribed non-antipsychotic medications or their combinations, $12.7 \%$ were taking one antipsychotic and $4.5 \%$ were prescribed more than one antipsychotic.

\section{Reported frequency of physical NCDs and associations with sociodemographic and clinical variables}

The overall reported frequency of any physical NCD was $24.9 \%$. Specifically, the frequency of diabetes mellitus, hypertension, chronic lung disease, heart disease, and kidney disease were $15.8 \%, 8.7 \%, 2.0 \%$, 1.5\% and $1 \%$, respectively. As the number of patients with self-reported presence of heart disease, lung disease or kidney disease was negligible, our analyses focused on patients with diabetes and/or hypertension. As shown in Table 2, 46.5\% of patients with diabetes had the condition for less than 5 years while $54.6 \%$ of patients with hypertension had the condition for at least 5 years or longer. More than $85 \%$ of patients with diabetes or hypertension were undergoing treatment for their physical NCD. Twenty patients (4.4\%) had both diabetes and hypertension.

Table 2. Prevalence of diabetes and hypertension, their treatment status and durationof treatment

\begin{tabular}{|l|l|}
\hline & $\mathbf{n}(\%)$ \\
\hline Diabetes & $71(15.8)$ \\
\hline $\begin{array}{l}\text { Duration of Diabetes } \\
<5 \text { years }\end{array}$ & $38(53.5)$ \\
$\geq 5$ years & $33(46.5)$ \\
\hline Patients undergoing treatment for Diabetes & $62(87.3)$ \\
Duration of Diabetes treatment & $32(51.6)$ \\
$<5$ years & $30(48.4)$ \\
$\geq 5$ years & $39(8.7)$ \\
\hline Hypertension & $16(41.0)$ \\
\hline Duration of Hypertension & $22(56.4)$ \\
\hline 55 years & $36(92.3)$ \\
$\geq 5$ years & $14(38.9)$ \\
\hline Patients undergoing treatment for Hypertension & $22(61.1)$ \\
\hline $\begin{array}{l}\text { Duration of Hypertension treatment } \\
<5 \text { years }\end{array}$ & $20(4.4)$ \\
\hline Patients with both Diabetes and Hypertension & \\
\hline
\end{tabular}


Age, presence of a family history of any physical NCD and duration of psychiatric illness of $\geq 5 y$ ears were significantly associated with the presence of diabetes or hypertension $(\mathrm{p}<0.05$, Table 3$)$.Schizophrenia, schizotypal and delusional disorders were most prevalent among patients with diabetes or hypertension, and combinations of antipsychotics with other psychotropic medications were predominantly prescribed to patients with these physical NCDs. No association was observed between the psychiatric diagnosis or psychotropic medications with the presence of diabetes or hypertension (Table 3).

Table 3. Association of sociodemographic and clinical variables with diabetes or hypertension status in patients with psychiatric illnesses

\begin{tabular}{|c|c|c|c|}
\hline Variable & $\begin{array}{c}\text { diabetes or } \\
\text { hypertension } \\
\mathrm{N}=90\end{array}$ & $\begin{array}{c}\text { no diabetes or } \\
\text { hypertension } \\
\mathrm{N}=360\end{array}$ & $\begin{array}{c}\text { p-value } \\
\text { (Chi- } \\
\text { squared test) }\end{array}$ \\
\hline Age, mean \pm SD (years) & $49.7 \pm 10.8$ & $38.6 \pm 11.5$ & $<0.0001^{*}$ \\
\hline Males & $33(36.7)$ & $165(45.8)$ & \\
\hline Females & $57(63.3)$ & $195(54.2)$ & 0.117 \\
\hline BMI, mean $\pm \mathrm{SD}\left(\mathrm{kg} / \mathrm{m}^{2}\right)^{\#}$ & $27.8 \pm 5.3$ & $27.1 \pm 5.0$ & 0.3220 \\
\hline Family history of any physical NCD & $69(76.7)$ & $216(60.0)$ & $0.003^{*}$ \\
\hline Family history of psychiatric illness & $25(27.8)$ & $122(33.9)$ & 0.269 \\
\hline $\begin{array}{l}\text { Duration of psychiatric illness } \\
<5 \text { years } \\
\geq 5 \text { years }\end{array}$ & $\begin{array}{l}25(27.8) \\
65(72.2) \\
\end{array}$ & $\begin{array}{l}145(40.3) \\
215(59.7)\end{array}$ & $0.029^{*}$ \\
\hline $\begin{array}{l}\text { Duration of psychiatric treatment } \\
<5 \text { years } \\
\geq 5 \text { years }\end{array}$ & $\begin{array}{l}52(57.8) \\
38(42.2)\end{array}$ & $\begin{array}{l}234(65.4) \\
124(34.6)\end{array}$ & 0.181 \\
\hline $\begin{array}{l}\text { Psychiatric diagnosis } \\
\text { Schizophrenia and related disorders } \\
\text { Mood (affective) disorders } \\
\text { Neurotic, and somatoform disorders } \\
\text { Others }\end{array}$ & $\begin{array}{c}51(56.7) \\
27(30.0) \\
6(6.7) \\
6(6.7) \\
\end{array}$ & $\begin{array}{c}\mathbf{0 . 1 1 8} \\
238(66.3) \\
68(18.9) \\
20(5.6) \\
33(9.2) \\
\end{array}$ & 0.118 \\
\hline $\begin{array}{l}\text { Psychotropic medications }^{€} \\
1 \text { antipsychotic } \\
>1 \text { antipsychotic } \\
\text { Combination of antipsychotics with other } \\
\text { medications } \\
\text { Other medication/combinations (no } \\
\text { antipsychotics) }\end{array}$ & $\begin{array}{l}10(11.1) \\
3(3.3) \\
61(67.8) \\
16(17.8)\end{array}$ & $\begin{array}{c}47(13.1) \\
17(4.7) \\
247(68.8) \\
48(13.4)\end{array}$ & 0.675 \\
\hline
\end{tabular}

(All data expressed as $\mathrm{n}(\%)$, unless otherwise indicated. ${ }^{\star}$ represents statistical significance of $\mathrm{p}<0.05$; \#Data missing from 139 patients; \$data missing from 2 patients; $€$ data missing from 1 patient)

\section{Role of the psychiatrist in managing physical NCDs}

In this study, we aimed to understand the role of the psychiatrist at an out-patient facility in managing patients' physical comorbidities either by themselves or by initiating referral to a general practitioner or specialist doctors. Less than $20 \%$ of patients with diabetes or hypertension were referred by the treating psychiatrist for further follow-up of the medical comorbidity. Only a small fraction of patients $(<8 \%)$ with diabetes or hypertension were treated for their physical NCD by the psychiatrist. Vast majority of patients with diabetes or hypertension in our study were undergoing treatment for these conditions at government or private hospitals/clinics. 
Based on BP measurements of patients who did not self-report the presence of hypertension, we observed that 40 patients $(11.1 \%$ of patients without diabetes or hypertension) had raised BP defined as $\geq 140$ and/ or $\geq 90 \mathrm{mmHg}$. These results suggest that this group of patients are at risk to develop hypertension or already have hypertension but are unaware and will need to be followed-up closely.

\section{DISCUSSION:}

This study is one amongst the few conducted in India to explore frequency of concurrent physical comorbidities such as diabetes, hypertension, chronic lung disease, chronic kidney disease or heart disease in a large cohort of 450 patients with a wide spectrum of psychiatric illnesses in an out-patient tertiary care setting, as elicited by a history . Approximately $25 \%$ of these patients selfreported the presence of at least one of these physical comorbidities. Diabetes was the most prevalent comorbidity, as reported by $15.8 \%$ of patients, followed by hypertension in $8.7 \%$ of patients. Psychiatrists at the tertiary care center played a limited role in management of these comorbid physical NCDs. Finally, the psychiatric out-patient facility has a potential for opportunistic screening for common physical NCDs such as diabetes or hypertension as 40 patients with elevated BP or at risk for hypertension were identified while conducting a physical examination as part of this study.

Other studies in India have found higher prevalence rates of medical comorbidity than the prevalence observed in our study.14-17,21Singh et al. conducted a study in a setting very similar to ours, except for the fact that clinical examination and investigations were conducted to identify various physical comorbidities. Close to half the patient population (48\%) had physical illnesses, with hypertension (29.1\%), respiratory diseases $(15 \%)$, and diabetes (10\%) amongst the most common diseases.16 Although not comparable, the difference in rates observed between our study and Singh et.al. could partly be explained by the fact that we depended on patient-reported presence of the illness(es), and not clinical investigations, thereby leading to under-reported rates. It is possible that the under-reporting could be due to lack of disease awareness, communication difficulties or hesitancy. In fact, previous studies have shown that psychiatric patients have difficulties in communicating their physical illnesses to the physician.22 Furthermore, it has been suggested that such difficulties are constant and not reduced in non-acute conditions.

In our study, majority of patients with diabetes or hypertension were diagnosed with schizophrenia, schizotypal and delusional disorders. It has been welldocumented that patients with schizophrenia suffer a high burden of diabetes and metabolic syndrome, and a higher prevalence compared to the general population.23 It is to be noted that an increase in well-known risk factors in these patients partially explains most of the increased risk. 4 In our study, too, we identified some of these risk factors such as age, family history of diabetes or hypertension and duration of psychiatric illness of 5 years or longer, to be associated with the presence of diabetes or hypertension.

Our study showed that less than $8 \%$ of patients were being treated for diabetes or hypertension at our tertiary care center, and less than $20 \%$ of patients were referred by a psychiatrist for further follow-up with respect to management of diabetes, hypertension or other physical illnesses. These results suggest that psychiatrists need to be more aware of management of common physical comorbidities such as diabetes and hypertension in patients with psychiatric illnesses. Patients often seek treatment for symptoms of disorders that are diagnosed as co-morbid, rather than principal conditions. As a complex interface exists between such physical and mental illnesses, psychiatrists need to be trained to treat patients with these common physical conditions, and in specific, severe mental illnesses. In this manner, improved screening and early treatment of physical illnesses in psychiatric patients can significantly impact their psychosocial functioning and quality of life.

Interestingly, through monitoring of BP during a patient's visit to our OP facility, we identified as many patients at risk of hypertension with elevated BP ( $\geq 140$ and/or $\geq 90$ $\mathrm{mmHg}$ ) as the number of patients with self-reported hypertension. In essence, this finding exemplifies the importance of regular physical examination and appropriate investigations at a tertiary psychiatric care center that in turn, can identify patients at risk of developing or already have diabetes, hypertension or MetS. Importantly, this observation highlights the potential of opportunistic screening of common physical NCDs in a psychiatric care center. In fact, a nurse-led intervention at a community mental health center resulted in 30\% more patients with severe mental illness who received screening for CVD risk factors such as blood pressure, cholesterol, glucose, BMI and smoking status.24This indicates that care coordination, or integrated care programs for physical health conditions in patients with mental illnesses can better-equip health systems and be more effective in reducing unfavourable health outcomes in these patients.

In the present study, the patients were recruited from a psychiatric out-patient facility. The findings of this study cannot be generalized to the entire population as these rates were based on patient-reported presence of the physical NCD and a wide-spectrum of psychiatric diagnoses were present in our study population. Further, we are not aware of the diagnostic criteria or tests used by the medical doctor to diagnose the patient with diabetes and/or hypertension. In this study, no laboratory diagnosis to confirm of the NCDs were done as the outpatient mental health clinic caters to patients who may not be able to afford laboratory expenses. Our findings 
need to be confirmed in larger community-based studies and also, by conducting clinical investigations to diagnose physical illnesses, especially in those who are at greater risk for cardiovascular disease. However, this study also demonstrates that a good clinical history for NCDs can provide valuable information to direct management of psychiatric patients, mental health professionals can be enabled to be aware of NCDs in patients and make appropriate referrals.

\section{CONCLUSION}

In conclusion, approximately $25 \%$ of patients visiting a tertiary psychiatric care center self-reported the presence of diabetes or hypertension. This study shows that physical examination andfamilial history can be helpful in detecting psychiatric patients at risk of developing physical NCDs in such a clinical setting. It is crucial for psychiatrists to increase their involvement in managing comorbid physical NCDs or for existing health systems to set up a referral path way to improve overall health outcomes for patients with psychiatric illnesses. Future studies are needed to identify potential differences that need to be incorporated while treating patients with physical NCDs based on the presence/absence of psychiatric illnesses. Studies are also required to develop a comprehensive psychosocial intervention strategy for patients with physical and mental comorbidities where lifestyle modifications are integrated within.

\section{ACKNOWLEDGEMENT}

We would like to thank Dr. Prabhdeep Kaur and Dr. Suresh Sankarasubbaiyan for their technical input during the course of the study and data analysis. We also thank Ms. Anjana Bala for helping us with preliminary data collection.

\section{SOURCE OF FUNDING: None}

\section{CONFLICT OF INTEREST}

The authors have declared no conflict of interest with respect to the research, authorship,and/or publication of this article

\section{REFERENCES}

1 Holt RIG andPeveler RC. Diabetes and cardiovascular risk in severe mental illness: a missed opportunity and challenge for the future. Practical Diabetes International. 2010;27(2):79-84

2 Roshanaei-Moghaddam B and Katon W. Premature mortality from general medical illnesses among persons with bipolar disorder: a review. Psychiatric Services. 2009;60(2):147-156

3 Laursen TM, Munk-Olsen T, Nordentoft M, et al. Increased mortality among patients admitted with major psychiatric disorders: a register-based study comparing mortality in unipolar depressive disorder, bipolar affective disorder, schizoaffective disorder, and schizophrenia. Journal of Clinical Psychiatry. 2007;68(6):899907

4 Parks J, Svendsen D, Singer P, et al. Morbidity and mortality in people with serious mental illness. Alexandria (Virginia): National Association of State Mental Health Program Directors (NASMHPD) Medical Directors Council. 2006 [cited 2018 May 25]. Available from: https://www.nasmhpd.org/content/morbidity-andmortality-people-serious-mental-illness.

5 De Hert M, Dekker JM, Wood D, et al. Cardio-vascular disease and diabetes in people with severe mental illness. Position statement from the European Psychiatric Association (EPA), supported by the European Association for the Study of Diabetes (EASD) and the European Society of Cardiology (ESC). European Psychiatry. 2009;24(6):412-424

6 Tiihonen J, Lönnqvist J, Wahlbeck K, et al. 11-year follow-up of mortality in patients with schizophrenia: a population-based cohort study (FIN11 study). Lancet. 2009;374(9690):620-627

7 Samele C, Patel M, Boydell J, et al. Physical illness and lifestyle risk factors in people with their first presentation of psychosis. Social Psychiatry and Psychiatric Epidemiology. 2007;42(2):117-124

8 Osborn DPJ, Nazareth I, King MB. Risk for coronary heart disease in people with severe mental illness: cross-sectional comparative study in primary care. British Journal of Psychiatry. 2006;188(3):271-277

9 Mitchell AJ, Malone D, Doebbeling CC. Quality of medical care for people with and without comorbid mental illness and substance misuse: systematic review of comparative studies. British Journal of Psychiatry. 2009;194(6):491-499 
10 Nasrallah HA, Meyer JM, Goff DC, et al. Low rates of treatment for hypertension, dyslipidemia and diabetes in schizophrenia: data from the CATIE schizophrenia trial sample at baseline. Schizophrenia Research. 2006;86(1-3):15-22

11 McIntyre RS, Soczynska JK, Beyer JL, et al. Medical comorbidity in bipolar disorder: re-prioritizing unmet needs. Current Opinion in Psychiatry. 2007;20(4):406-416

12 Rummel-Kluge C, Komossa K, Schwarz S, et al. Head-to-head comparisons of metabolic side effects of second-generation antipsychotics in the treatment of schizophrenia: a systematic review and meta-analysis. Schizophrenia Research. 2010;123(2-3):225-233

13 Leucht S, Corves C, Arbter D, et al. Second-generation versus first-generation antipsychotic drugs for schizophrenia: a meta-analysis. Lancet. 2009;373(9657):31-41

14 Mattoo SK and Singh SM. Prevalence of metabolic syndrome in psychiatric inpatients in a tertiary care centre in north India. Indian Journal of Medical Research. 2010;131(1):46-52

15 Hussain T, Margoob MA, Shoib S, et al. Prevalence of Metabolic Syndrome among Psychiatric Inpatients: A Hospital Based Study from Kashmir. Journal of Clinical and DiagnosticResearch. 2017;11(6):VC05-VC08

16 Singh GP, Chavan BS, Kaur P, Bhatia S. Physical illnesses among psychiatric outpatients in a tertiary care health institution: a prospective study. Indian Journal of Psychiatry. 2006;48(1):52-55

17 Das D, Bora K, Baruah B, et al. Prevalence and predictors of metabolic syndrome in schizophrenia patients from Assam. Indian Journal of Psychiatry. 2017;59(2):228-232

18 Naing L, Winn T, Rusli BN. Practical Issues in Calculating the Sample Size for Prevalence Studies. Archives of Orofacial Sciences.2006;1:9-14

19 World Health Organization. WHO STEPwise approach to Surveillance (STEPS). Geneva (Switzerland). 2015 [cited 2018 May 25]. Available from:http://www.who.int/chp/steps /en

20 World Health Organization. ICD-10 Version: 2016. Geneva (Switzerland). 2016 [cited 2018 May 25]. Available from: http://apps.who.int/classifications/icd10/browse/2016/en.

21 Manuel CM, Rao PP, Rebello P, et al. Medical comorbidity in in-patients with psychiatric disorder. Muller Journal of Medical Sciences and Research. 2013;4(1):12-17

22 Pary RJ and Barton SN. Communication difficulty of patients with schizophrenia and physical illness. Southern Medical Journal. 1988;81(4):489-490

23 DE Hert M, Correll CU, Bobes J, et al. Physical illness in patients with severe mental disorders. I. Prevalence, impact of medications and disparities in health care. World Psychiatry 2011;10(1):52-77

24 Osborn DP, Nazareth I, Wright CA, et al. Impact of a nurse-led intervention to improve screening for cardiovascular risk factors in people with severe mental illnesses. Phase-two cluster randomized feasibility trial of community mental health teams. BMC Health Services Research. 2010;10:61 